ARTICLE

\title{
Synthetic biology and microbioreactor platforms for programmable production of biologics at the point-of-care
}

Pablo Perez-Pinera ${ }^{1,2, *, \dagger}$, Ningren Han ${ }^{2, \star}$, Sara Cleto ${ }^{1,2}$, Jicong Cao ${ }^{1,2}$, Oliver Purcell ${ }^{1,2}$, Kartik A. Shah $^{3}$, Kevin Lee ${ }^{4}$, Rajeev Ram² \& Timothy K. Lu, ${ }^{1,2,5}$

Current biopharmaceutical manufacturing systems are not compatible with portable or distributed production of biologics, as they typically require the development of single biologic-producing cell lines followed by their cultivation at very large scales. Therefore, it remains challenging to treat patients in short time frames, especially in remote locations with limited infrastructure. To overcome these barriers, we developed a platform using genetically engineered Pichia pastoris strains designed to secrete multiple proteins on programmable cues in an integrated, benchtop, millilitre-scale microfluidic device. We use this platform for rapid and switchable production of two biologics from a single yeast strain as specified by the operator. Our results demonstrate selectable and near-single-dose production of these biologics in $<24 \mathrm{~h}$ with limited infrastructure requirements. We envision that combining this system with analytical, purification and polishing technologies could lead to a small-scale, portable and fully integrated personal biomanufacturing platform that could advance disease treatment at point-of-care.

\footnotetext{
${ }^{1}$ Synthetic Biology Group, Department of Biological Engineering and Electrical Engineering \& Computer Science, Massachusetts Institute of Technology, Cambridge, Massachusetts 02142, USA. ${ }^{2}$ Research Laboratory of Electronics, Massachusetts Institute of Technology, Cambridge, Massachusetts 02142, USA. ${ }^{3}$ The David H. Koch Institute for Integrative Cancer Research, Massachusetts Institute of Technology, Cambridge, Massachusetts 02139, USA.

${ }^{4}$ Pharyx Inc., Woburn, Massachusetts 01801, USA. ${ }^{5}$ The Broad Institute of MIT and Harvard, Cambridge, Massachusetts 02412, USA. * These authors contributed equally to this work. † Present address: Department of Bioengineering, University of Illinois at Urbana-Champaign, Urbana, Illinois 61801, USA. Correspondence and requests for materials should be addressed to T.K.L. (email: tim@lugroup.org).
} 
Diopharmaceutical production is one of the largest contributors to escalating expenditures in healthcare, in particular due to the length, complexity and high costs associated with product discovery and development as well as the cost of construction and scale-up of biomanufacturing ${ }^{1-3}$. In addition, biologics treatment of patients in remote parts of the world, battlefields, emergency situations or under-developed regions with poor infrastructure presents formidable logistical challenges that greatly impact drug availability at the point-ofcare $^{4-9}$.

Currently, manufacturing of biologic drugs in the biopharmaceutical industry relies heavily on large-scale fermentation batches that are frequently monitored offline, to ensure a robust process and consistent quality of product ${ }^{4,8,10}$. However, as personalized medicines, single-use technologies and the desire for global and decentralized access to biologics are becoming increasingly important, there is a growing need for rapid, flexible, scalable and portable biomanufacturing systems that can be monitored/controlled online for affordable, safe and consistent production of biologics ${ }^{11}$.

To address this demand for personalized biomanufacturing technologies, we developed a new platform for flexible and portable production of biologic therapeutics at the point-of-care in short time frames and with limited system requirements. The basic components of this platform are as follows: (1) a biologics expression system engineered to secrete multiple therapeutic proteins in response to programmable cues and (2) an integrated, small overall footprint, millilitre-scale perfusion microfluidic platform capable of supporting rapid and flexible biomanufacturing process control that enables portable operations in mobile units, such as vehicles (Fig. 1 and Supplementary Movie 1). We demonstrate that this system produces near-single-dose levels of recombinant human growth hormone $(\mathrm{rHGH})$ and interferon- $\alpha 2 \mathrm{~b}$ (IFN $\alpha 2 \mathrm{~b}$ ) in $<24 \mathrm{~h}$.

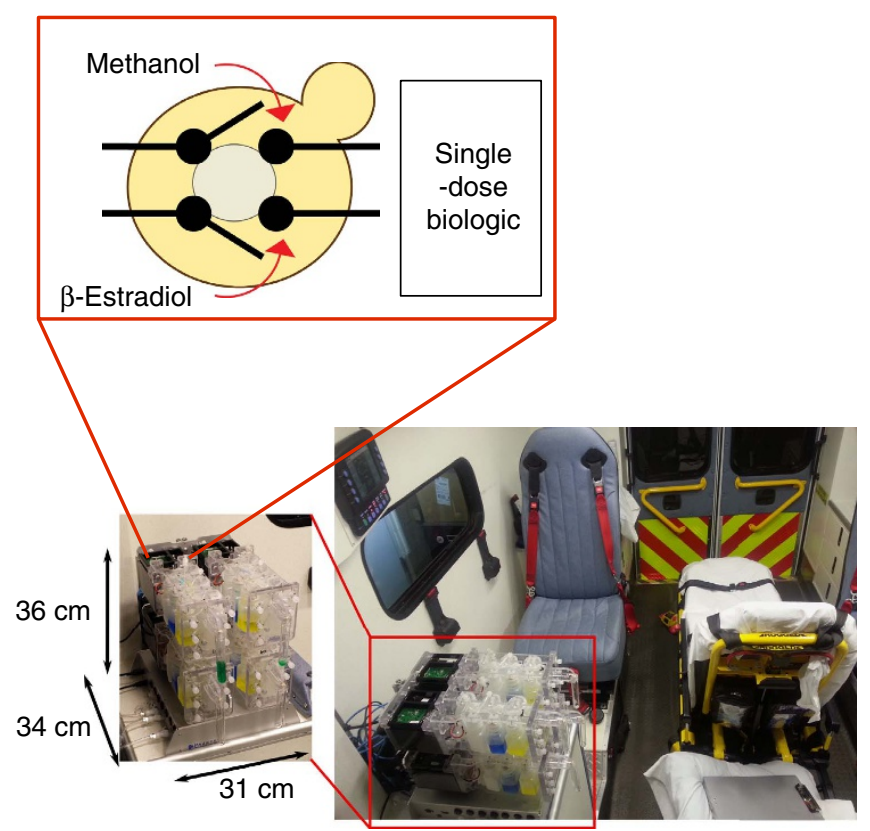

Figure 1 | Point-of-care biomanufacturing by integrating genetically engineered strains and portable microbioreactors for programmable biologics expression. $P$. pastoris strains genetically engineered to contain independently controllable inducible genetic cassettes were used within portable perfusion microbioreactors to achieve high-density and programmable expression of two biologic drugs. Here we show the microbioreactor system in the context of an ambulance.

\section{Results}

Host selection. Recombinant biologics for therapeutic use in humans can be produced using a variety of host organisms, including bacteria, yeast, plants, insect cells and mammalian cells $^{12}$. The specific host used can have an impact on yields, need for viral inactivation, downstream purification requirements, as well as final product formulation. Mammalian Chinese Hamster Ovary (CHO) cells are the most commonly used host for producing Food and Drug Administration (FDA)-approved biologics 9 , but they have complex media requirements and their storage requires cryopreservation. Therefore, the long time needed to go from inoculation of biologic-producing $\mathrm{CHO}$ cells to release of a drug product, which meets established quality standards and an FDA-approved safety profile, is incompatible with a rapid production system. Yeast are attractive alternatives to $\mathrm{CHO}$ cells, as they have simple media requirements, grow quickly to high densities and can be stored as lyophilized material ${ }^{12,13}$. Pichia pastoris (also known as Komagataella phaffi) is becoming increasingly popular for biologic production, as it (1) can grow to very high densities on simple and inexpensive carbon sources; (2) has a strong yet tightly controlled alcohol oxidase 1 (AOX1) promoter, which can be induced by methanol for high level protein production (up to $10 \mathrm{gl}^{-1}$ ) and is effectively repressed by glycerol or glucose; (3) is capable of human-like posttranslational modifications, including glycosylation ${ }^{14-16}$; and (4) secretes heterologous proteins into the extracellular space very efficiently with minimal host protein contamination, thus requiring relatively simple downstream purification systems ${ }^{17,18}$.

To date, more than 500 different proteins, including simple peptides, enzymes, hormones, monoclonal antibodies and FDA-approved therapeutics have been expressed in $P$. pastoris ${ }^{1,4,5}$. Thus, as a proof-of-concept of flexible biologics manufacturing using a single host, we developed strains of $P$. pastoris to support the independently selectable production of two different therapeutics. The use of individual strains that support production of multiple biologics provides significant advantages over the use of multiple strains that each produce single biologics. First, leveraging strains that produce multiple biologics for rapid production of dosage-level biologics enables the re-use of accumulated biomass from the outgrowth period. This approach dramatically improves production speed by avoiding the need to regrow different strains into production-level biomass. Second, for production of multicomponent products, the FDA requires approval of multiple manufacturing lines that each produce individual components and thus a single multiplexed expression platform could offer a potential regulatory advantage.

The AOX1 promoter $\left(\mathrm{P}_{\mathrm{AOX} 1}\right)$ is useful for producing one protein on-demand. Only a few alternative inducible promoters have been characterized in $P$. pastoris, including the CUP1, G1 or FLD1 promoters. However, it remains unclear whether these promoters support high levels of expression, while remaining orthogonal to $\mathrm{P}_{\text {AOX1 }}$ (refs 19-21). Thus, to enable selectable bioproduction, we sought to develop new inducible promoters that are orthogonal to and can surpass $\mathrm{P}_{\mathrm{AOX} 1}$ in promoter strength.

Transformation platform. Current transformation methods for $P$. pastoris rely on genomic integration of small linearized plasmids through homologous recombination, followed by antibiotic selection and screening for high-copy-number integrants ${ }^{22}$. Although these approaches are adequate for simple genetic manipulations, such as introducing small expression cassettes, they have several limitations for more sophisticated synthetic biology applications. In addition, multiple random integration events are undesirable when attempting to compare expression levels between different genetic constructs. 
To accelerate the rapid design-test-and-optimize cycle for creating new promoters, we first sought to overcome the ratelimiting step of plasmid transformation and genomic integration of synthetic constructs into $P$. pastoris. We created a recombinase-based system for the single-copy integration of plasmids at defined loci that is suitable even for large DNA constructs (Fig. 2a). We first generated a parent $P$. pastoris strain containing $a t t B$ sites for the recombinases BxbI, R4 and TP-901 (ref. 23). This was accomplished by traditional integration of a construct containing regions of homology to the Trp2 locus in the $P$. pastoris genome (Supplementary Tables 1 and 2, Integration Site 1), attB sites and a KanR selection cassette. Integration at the Trp2 locus was validated using PCR and a copy number of 1 was verified using quantitative PCR (qPCR; Supplementary Fig. 1). We then co-transformed a plasmid for the transient expression of BxbI, R4 or TP901, together with a transfer plasmid containing attP sites for the corresponding recombinase and engineered genetic constructs of interest. This method typically generated $\sim 50-300$ transformants per reaction for DNA constructs ranging from $\sim 7.8$ to $13.6 \mathrm{~kb}$ (Supplementary Fig. 2).

Optimization of $\beta$-estradiol-inducible expression systems. Using this integration strategy, we implemented an inducible transcriptional system consisting of a constitutively expressed zinc-finger (ZF) DNA-binding domain ${ }^{24}$ fused with the $\beta$-estradiol binding domain of the human oestrogen receptor, which is coupled with a transcriptional activation domain ${ }^{25}$. At steady state, this synthetic ZF transcription factor (ZF-TF) is sequestered in the cytoplasm by HSP90. Addition of $\beta$-estradiol displaces HSP90 and permits translocation of the ZF-TF into the nucleus, where it activates expression of genes regulated by a minimal promoter placed downstream of multiple ZF-binding sites (Fig. 2b). This system offers a highly flexible architecture that can be tuned by modifying different parameters, including the following: (1) affinity of the DNA-binding domain to DNA; (2) strength of the transcriptional activation domain; (3) number of binding sites for the ZF; (4) promoter driving expression of the ZF; (5) minimal promoter driving expression of the output; (6) dose of inducer; and (7) integration site.

Initial tuning of expression levels was performed with green fluorescent protein (GFP) as the output quantified using flow cytometry. The ZF DNA-binding domain used in these experiments was ZF43-8 (ref. 24). We fused the ZF DNA-binding domain and $\beta$-estradiol-binding domain of the human oestrogen receptor to the VP64 transcriptional activation domain, which has been previously shown to mediate higher levels of expression in mammalian cells than other domains, such as p65 or VP16 (ref. 26). The Saccharomyces cerevisiae TEF1 promoter was used to express the ZF-TF and a minimal GAP promoter preceded by nine binding sites for ZF43-8 was used to drive the inducible expression of GFP. At $24 \mathrm{~h}$, dose-response curves showed that maximum expression of GFP could be attained with only $0.01 \mu \mathrm{M}$ $\beta$-estradiol (Fig. 2c). However, at $48 \mathrm{~h}, 0.1-1 \mu \mathrm{M} \beta$-estradiol was necessary to fully saturate this system.

We further optimized gene expression by modulating the number and placement of ZF-binding sites within the artificial promoter, which have been shown to be important parameters for maximizing the expression of regulated genes ${ }^{27}$. We used a ppTEF1 promoter to express the ZF-TF, which targeted a minimal CYC promoter preceded by single ZF-binding sites placed $\sim 200, \sim 350$ or $\sim 500$ upstream of the ATG start codon, as well as combinations of two or three ZF-binding sites. We also tested one promoter with nine binding sites spaced $\sim 20 \mathrm{bp}$ and one promoter with nine ZF-binding sites spaced $\sim 40 \mathrm{bp}$ from each other (with the closest ZF-binding site located $200 \mathrm{bp}$ upstream of the ATG codon of the output gene). We found that promoters with nine binding sites expressed GFP at higher levels than promoters with three binding sites when induced, whereas increasing the spacing between binding sites did not significantly improve levels of expression (Fig. 3).

Chromosomal context is an important factor to consider when expressing heterologous genes, because promoter interference, chromatin structure or other epigenetic modifications can have a negative impact on gene activation ${ }^{28,29}$. Thus, we tested GFP expression from cassettes integrated at nine different chromosomal loci. These loci correspond to regions targeted in prior reports ${ }^{22,30}$ or to intergenic regions, to avoid directly disrupting native coding sequences (Supplementary Tables 1 and 2). We did not observe statistically significant differences in the maximally induced levels of gene expression across nine loci (Supplementary Fig. 3).

The performance of the ZF-inducible expression system can be further affected by two additional parameters: the strength of the promoter driving expression of the ZF-TF and the basal activity of the minimal promoter, which contains ZF-binding sites, that controls expression of the output. The promoter driving ZF-TF expression is constitutive and determines how much ZF-TF accumulates in the cytoplasm. Excessive ZF-TF levels can potentially surpass the capacity of HSP90 to sequester the ZF-TF in the cytoplasm and, as a result, the ZF-TF may spontaneously translocate into the nucleus and activate expression in the absence of inducer, thus increasing undesired background. To minimize background expression levels, the minimal promoter containing ZF-binding sites should activate gene expression only when the inducer is present.

To fine-tune the expression of our output signal, we expressed the ZF-TF from several constitutive promoters previously used in $P$. pastoris, as well as variations of the GAP promoter that we constructed by introducing $\sim 50 \mathrm{bp}$ insertions throughout its DNA sequence to modify its activity (Supplementary Fig. 4). We built combinations of these ZF-TF-expressing constitutive promoters with minimal promoters derived from those that regulate AOX1, GAP, a GAP6 variant, GCW14 and S. cerevisiae CYC1 (Fig. 4). We measured GFP expression via flow cytometry with and without induction with $\beta$-estradiol. The different combinations exhibited a wide range of background expression levels and maximal activation ratios (Fig. 4a). Interestingly, some of the combinations consistently supported maximum expression levels higher than those reached by GFP expressed directly from the well-characterized $\mathrm{P}_{\mathrm{AOX} 1}$ under maximal induction (Supplementary Fig. 5). Overall, combinations that exhibited higher maximum expression levels also had greater background levels in the OFF state.

We selected three architectures that had ON/OFF ratios in excess of $\sim 4$-fold and/or high maximal expression activities for further engineering (architectures 245, 246 and 255; Fig. 4b). We then added an expression cassette to produce red fluorescent protein (RFP) using the AOX1 promoter to these vectors and generated strains $245 \mathrm{R}, 246 \mathrm{R}$ and $255 \mathrm{R}$, respectively (Fig. 5a). As expected, these strains expressed GFP when induced with $\beta$-estradiol in buffered glycerol-complex medium (BMGY), expressed RFP when induced with buffered methanol-complex medium (BMMY) and expressed both GFP and RFP when induced with $\beta$-estradiol in BMMY (Fig. 5b).

Using the synthetic expression cassettes that were optimized with intracellular fluorescent reporters 245R, 246R and 255R (Fig. 5b), we then built a proof-of-concept system to controllably produce two biologic drugs, $\mathrm{rHGH}$ and IFN $\alpha 2 \mathrm{~b}$. This resulted in three strains capable of selectable expression of two different biologics: strain 245B (GAP promoter expressing ZF-TF, AOX1 minimal promoter with ZF-binding sites expressing $\mathrm{rHGH}$ 
a

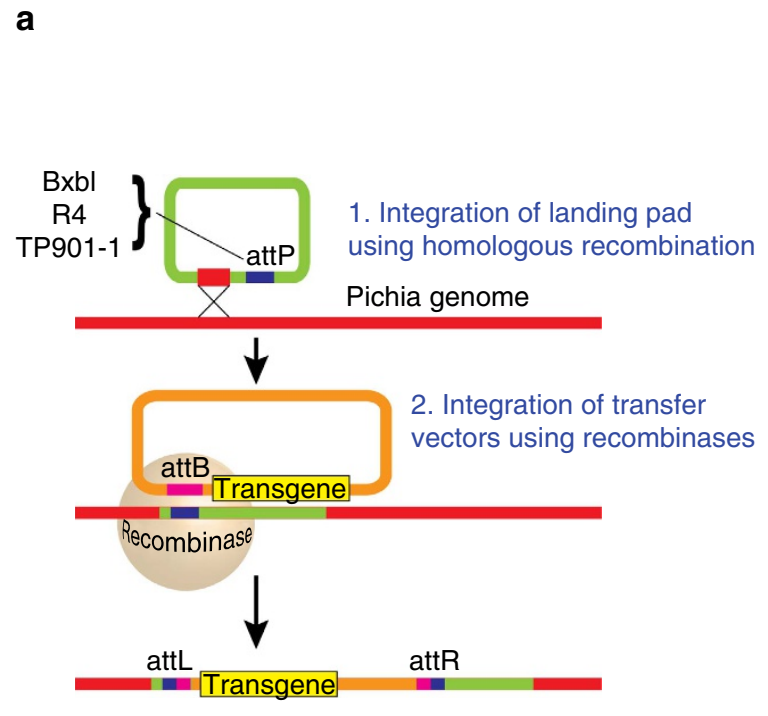

C

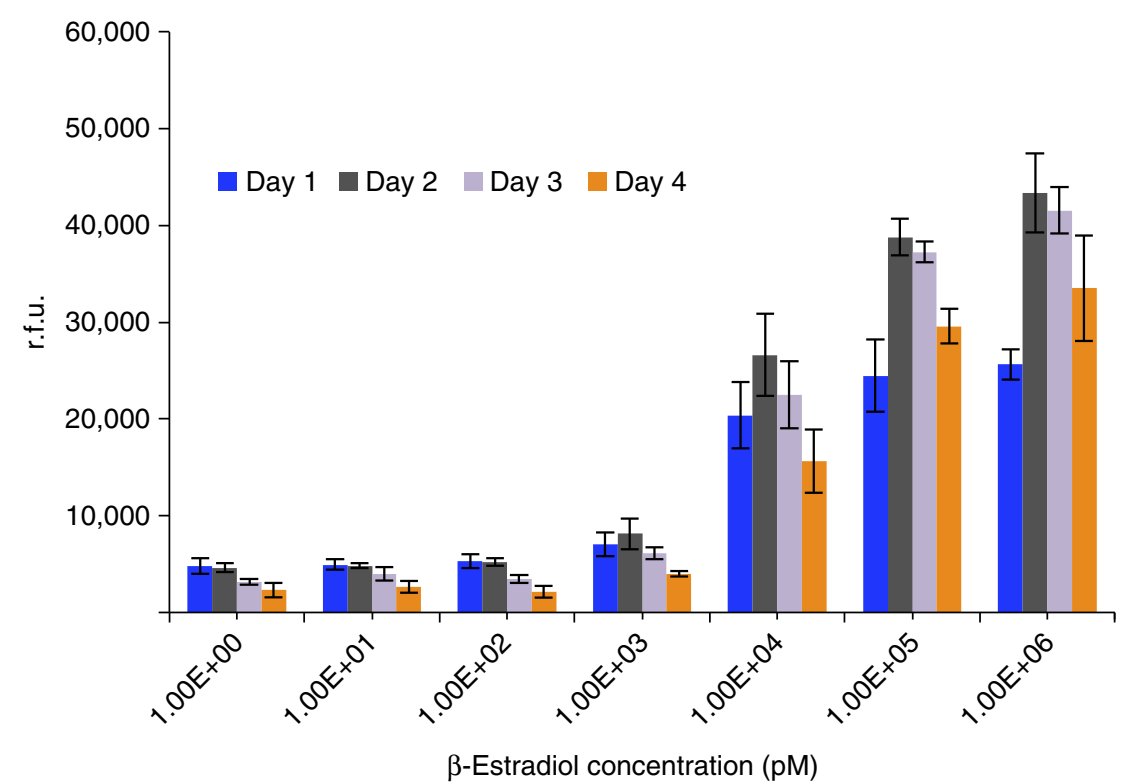

b

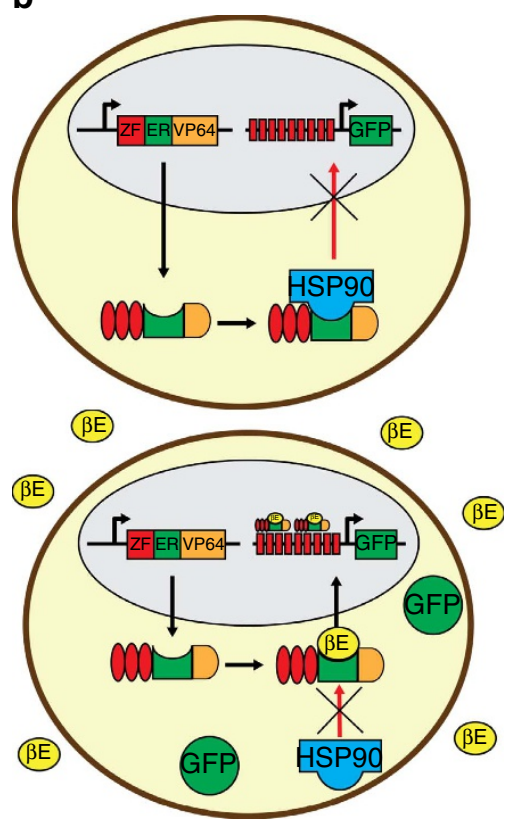

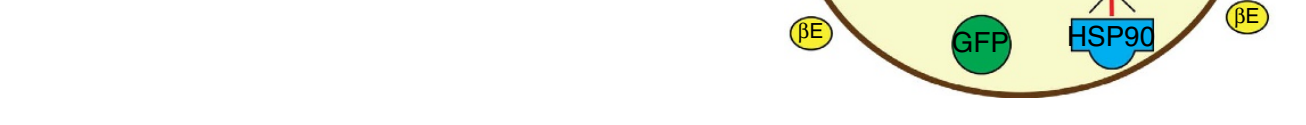

$\beta$-Estradiol concentration $(\mathrm{pM})$

Figure 2 | Development of artificial promoter systems for high-level transgene expression in P. pastoris. (a) Schematic representation of the landing-pad-based integration system used in these studies. We first generated a parental strain containing landing pads based on attB sites for the recombinases Bxbl, R4 and TP-901.1. This strain can be efficiently transformed with a transfer vector containing the desired gene circuit and the corresponding attP site together with a plasmid encoding the corresponding recombinase. (b) Schematic representation of the $\beta$-estradiol-inducible system used for in this study. This system uses a ZF DNA-binding domain fused to the $\beta$-estradiol-binding domain of the human oestrogen receptor, which is coupled to a transcriptional activation domain. This synthetic ZF-TF is sequestered in the cytoplasm by HSP90. Addition of $\beta$-estradiol displaces HSP9O and permits translocation of the ZF-TF into the nucleus, where it activates expression of genes regulated by a minimal promoter placed downstream of multiple ZF-binding sites. (c) Dose-response and time course of GFP expression using the $\beta$-estradiol-inducible system, where the S. cerevisiae TEF1 promoter was used to express the ZF-TF, and a minimal GAP promoter preceded by nine binding sites of ZF43-8 was used for inducible expression of GFP. $\beta$-Estradiol $(0.1-1 \mu \mathrm{M})$ is necessary to achieve full activation of this system at $48 \mathrm{~h}$. Error bars represent s.e.m. $(n=3)$.

and AOX1 promoter expressing IFN $\alpha 2 \mathrm{~b}$ ), strain 246B (GAP6 promoter expressing ZF-TF, AOX1 minimal promoter with ZF-binding sites expressing $\mathrm{rHGH}$ and AOX1 promoter expressing IFN $\alpha 2 \mathrm{~b}$ ) and strain 255B (scTEF1 promoter expressing ZF-TF, GAP minimal promoter with ZF-binding sites expressing rHGH and AOX1 promoter expressing IFN $\alpha 2 \mathrm{~b}$ ). Strain 245B, $246 \mathrm{~B}$ and $255 \mathrm{~B}$ were capable of selectable production of high-dose rHGH or IFN $\alpha 2 \mathrm{~b}$ in shake flasks within $24 \mathrm{~h}$ of induction (Fig. 5c).

Interestingly, the level of protein secretion was similar from the AOX1 promoter or the $\beta$-estradiol-inducible system when $\mathrm{rHGH}$ expression was induced after $48 \mathrm{~h}$ of outgrowth (Supplementary Fig. 6). One difference between the systems is that $\beta$-estradiol can be used to induce protein secretion without 


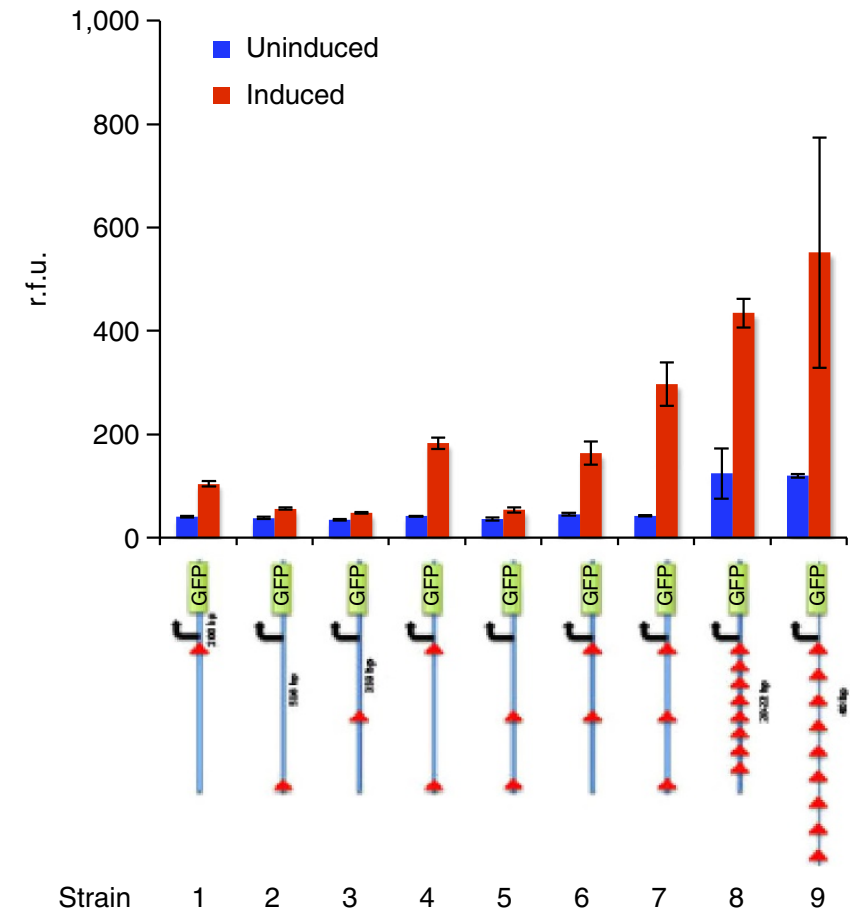

Figure 3 | Optimization of ZF-binding sites (triangles) in the minimal promoter for estradiol-inducible expression. Different combinations of three ZF-binding sites were placed $\sim 200, \sim 350$ or $\sim 500$ bp from the ATG, as well as binding sites spaced $\sim 20$ or $\sim 40$ bp from each other. The results show that more ZF-binding sites yield higher levels of GFP expression, whereas changing the spacing between binding sites did not significantly improve levels of expression. Error bars represent s.e.m. $(n=3)$.

outgrowth, while using glycerol as a carbon source, thereby allowing growth and production to occur simultaneously. Methanol induction, however, requires biomass accumulation (typically in glycerol) before the induction phase ${ }^{22}$. Without prior biomass accumulation, protein production from AOX1 was significantly lower than production from a $\beta$-estradiol-inducible promoter (Supplementary Fig. 7). As the daily dose of $\mathrm{rHGH}$ needed to treat patients is higher than the dose of IFN $\alpha 2 b$, we engineered $P$. pastoris strains for expression of $\mathrm{rHGH}$ on $\beta$-estradiol stimulation and expression of IFN $\alpha 2 \mathrm{~b}$ on methanol stimulation. To maximize protein production, we further tested different media additives and determined that formulations containing the antifoams L81, P2000 and AF204 enhanced rHGH secretion levels from strain 255, where GFP was replaced with rHGH (Supplementary Fig. 8).

Continuous and selectable protein production in microbioreactors. Sequential manufacturing of multiple biological therapeutics from individual strains requires rapid changes to their environment in high-density cell cultures. Furthermore, manufacturing at the volume scale of individual doses (for example, millilitres) suits requirements for point-of-care applications. For example, a production yield of $120 \mu \mathrm{g} \mathrm{ml}^{-1}$ for IFN $\alpha 2 b$ in yeast ${ }^{31}$ could result in multiple doses when produced at the millilitre scale, as a common formulation of IFN $\alpha 2 b$ (Intron-A, db00105) is $11.6 \mu \mathrm{g}$ (ref. 32). These two requirements can be complementary as the large surface-area-to-volume ratio afforded by miniature systems facilitates rapid media changeover. To demonstrate this, we developed a protocol specifically tailored for programmable protein production with our engineered strains in an integrated, millilitre-scale table-top microbioreactor that can be operated continuously for point-of-care use in personal biomanufacturing, even with limited resources. By combining our engineered dual-biologics strain with the operational flexibility of the microbioreactor device, we extended the traditional biomanufacturing approach, where a single biologic is produced per process, into one that enables sequential or controllable expression of multiple different biologics.

The principal component of the microbioreactor is a polycarbonate-Polydimethylsiloxane (PDMS) membrane-polycarbonate sandwiched chip with active microfluidic circuits outfitted pneumatically for routing of reagents, precise peristaltic injection, growth chamber mixing and fluid extraction (Fig. 6a) $)^{33}$. We used an injection volume of $700 \sim 900 \mathrm{nl}$ per injection for precise control of fluid addition/extraction in the 1-ml volume growth chamber. A $0.8-\mu \mathrm{m}$ pore size perfusion filter (polyethersulfone) with a $1-\mathrm{cm}$ diameter was incorporated underneath the growth chamber, to allow for fluid flow-through while maintaining all of the cells inside the growth chamber and enabling the switching of induction media (Fig. 6a). The ratio of the filter surface area to bioreactor volume was 0.758 - a factor of 3 higher than high-performance, bench-scale perfusion bioreactors previously reported ${ }^{34}$.

To demonstrate the paradigm of personalized, single-dose and programmable biomanufacturing, we performed 3-day continuous cultivation experiments for selectable production of two biologics at near-single-dose levels in $<24 \mathrm{~h}$. The dualbiologics-producing $P$. pastoris strain $255 \mathrm{~B}$ was inoculated from a single colony and grown in BMGY, first in batch and then in perfusion mode, with a perfusion rate of $0.5 \mathrm{ml} \mathrm{h}^{-1}$. At $24 \mathrm{~h}$, the outgrowth media was switched to our custom methanol media with perfusion rates of $1 \mathrm{ml} \mathrm{h}^{-1}$ for $4 \mathrm{~h}$ for rapid changeover of the chemical environment, followed by IFN $\alpha 2 \mathrm{~b}$ collection at a perfusion rate of $0.5 \mathrm{ml} \mathrm{h}^{-1}$ for $20 \mathrm{~h}$. The $1 \mathrm{ml} \mathrm{h}^{-1}$ changeover rate was chosen to ensure that $>98 \%$ of the preceding medium had been flushed out after this 4 -h medium changeover period according to our rate equation model for microbioreactor operation (see 'Microbioreactor flow modelling' in Methods). After the 48-h time point, we switched the custom methanol media with the custom $\beta$-estradiol-containing media at a perfusion rate of $1 \mathrm{ml} \mathrm{h}^{-1}$ for $4 \mathrm{~h}$, followed by a collection phase for $\mathrm{rHGH}$ lasting $20 \mathrm{~h}$ at a perfusion rate of $0.5 \mathrm{mlh}^{-1}$. A summary of the microbioreactor control operation is provided in Table 1. The different operational phases are illustrated with the online optical density (OD) plot in Fig. 6b. This experimental procedure resulted in $10 \mathrm{ml}$ of perfusate for each protein production period lasting $20 \mathrm{~h}$ each.

During the cultivation, cells inside the growth chamber were rapidly circulated and mixed by peristalsis. The fermentation temperature was controlled at $30 \pm 0.1^{\circ} \mathrm{C}$. The online OD was recorded through an optical path length of $\sim 250 \mu \mathrm{m}$ by a $630-\mathrm{nm}$ light-emitting diode, where the optical path length is chosen to maximize the linear response range compatible with our fabrication process. The dissolved oxygen level of the culture was monitored online and controlled by dynamically changing the gas feed line between air and oxygen to match the dissolved oxygen set point, which was set to $100 \%$ air saturation in the experiment. The online $\mathrm{pH}$ data were also recorded during the fermentation process. The real-time sensor data for the microbioreactor experiments presented here can be found in the Supplementary Fig. 9. A video showing the peristaltic mixing and optical sensing in the microbioreactor during the culture is provided in Supplementary Movie 2. We used four different input ports for the injection of BMGY outgrowth media, custom-made methanol media for IFN $\alpha 2 b$ production, $\beta$-estradiol-containing media for $\mathrm{rHGH}$ production and water for evaporation 


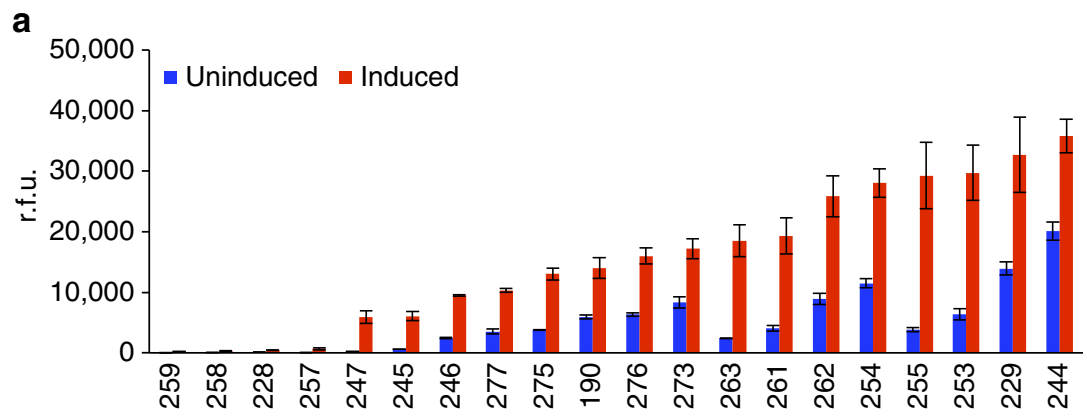

b

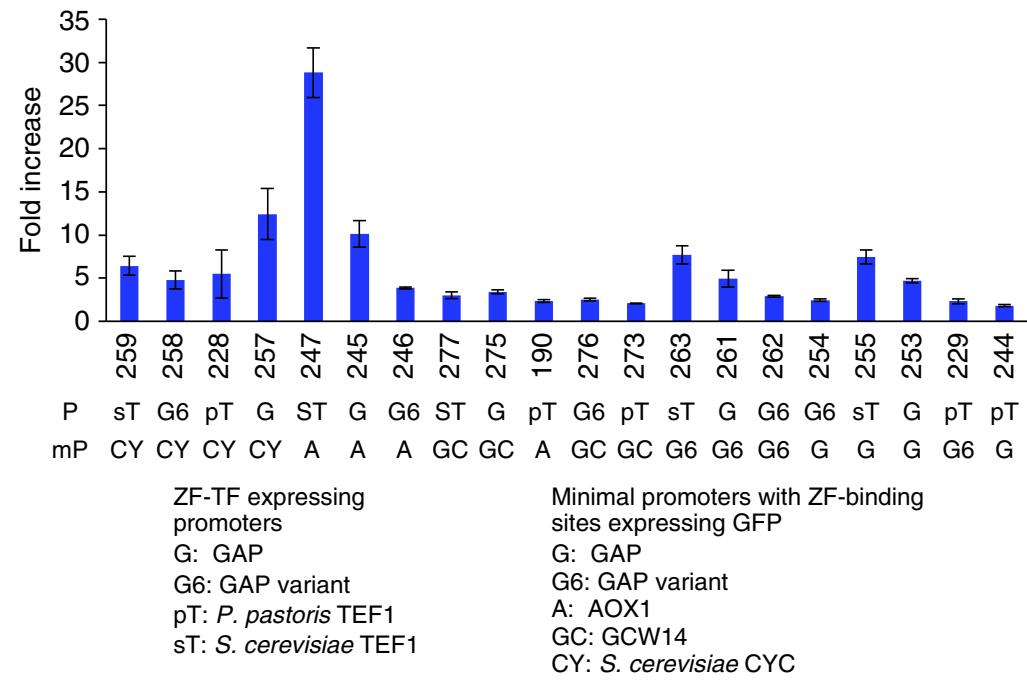

Figure 4 | Promoter optimization for high-level estradiol-inducible expression. (a) Four different promoters were used to express the ZF-TF, including the GAP promoter, a variant GAP promoter and the TEF1 promoter from P. pastoris, as well as the S. cerevisiae TEF1 promoter. We used five different minimal promoters containing nine ZF-binding sites located upstream of the ATG codon that are targeted by the ZF-TF to control GFP expression. The different combinations exhibited a wide range of background expression and maximal activation after $24 \mathrm{~h}$ of induction with $\beta$-estradiol in BMGY. Error bars represent s.e.m. $(n=3)$. (b) Fold increase in levels of GFP expression after induction with estradiol. Error bars represent s.e.m. $(n=3)$.

compensation. Perfusate was collected through the fluid channel downstream of the perfusion filter for protein characterization. For portable storage operation where lyophilized material may be used, the lyophilized material can be re-suspended in the reconstitution media before inoculation. This can serve as the seed inoculum and be injected into the growth chamber during the inoculation process. The cells could be revived inside the growth chamber subsequently without the need of additional steps. If the reconstitution media differs from the outgrowth media, a perfusion media changeover to the outgrowth media can be performed after the revival process.

To understand induction dynamics and protein secretion levels, we collected samples every hour during the 4-h media switching periods. In addition, during the 20 - $\mathrm{h}$ production periods, we first collected four samples every $2.5 \mathrm{~h}$ and then one sample after the last $10 \mathrm{~h}$. A standard enzyme-linked immunosorbent assay (ELISA) was carried out to quantify protein production. As shown in Fig. 6c, production profiles for the two biologics corresponded to the different induction media toggled by the microbioreactor. Four parallel microbioreactor experiments were carried out at the same time with identical protocols. Induction with methanol resulted in the rapid secretion of IFN $\alpha 2 \mathrm{~b}$, which reached maximal productivity after only $3 \mathrm{~h}$ and remained constant during the production period, and then rapidly decreased after methanol was removed from the media. Similarly, rHGH secretion was induced via media changeover to the custom $\beta$-estradiol-containing media, thus demonstrating multi-product expression control in our integrated platform.

A summary of cumulative protein production and measurements of wet cell weight at the end of the experiment averaged across the four microbioreactors is shown in Table 2. The total average production of IFN $\alpha 2 \mathrm{~b}$ was $19.73 \mu \mathrm{g}$ per reactor, which exceeds the $11.6 \mu \mathrm{g}$ dose in Intron-A, whereas the total average production of $\mathrm{rHGH}$ was $43.7 \mu \mathrm{g}$ per reactor (a common starting weight-based formulation of $\mathrm{rHGH}$ (Nutropin) is $0.006 \mathrm{mg} \mathrm{kg}^{-1}$ per $\left.\mathrm{day}^{35}\right)$. Therefore, even without extensive bioprocess optimization, this system is capable of producing IFN $\alpha 2 b$ in excess of the daily dose needed in adults and matching the daily dose $\mathrm{rHGH}$ required to treat infants. Importantly, the cultivation conditions in the perfusion microbioreactor provided continuous nutrient supplies and high oxygen transfer rates ${ }^{33}$ that led to the highest reported cell culture density achieved in any microfluidic platform, measured as an average wet cell weight of $356 \pm 27 \mathrm{gl}^{-1}$ (ref. 36). In addition to the microbioreactor run shown in Fig. 6, three additional microbioreactor runs operating the same protocol were carried out. The additional results are shown in Supplementary Fig. 10.

\section{Discussion}

The choice of therapeutic molecules and the regulatory gene circuits used in a multiplexed biomanufacturing platform is 
a
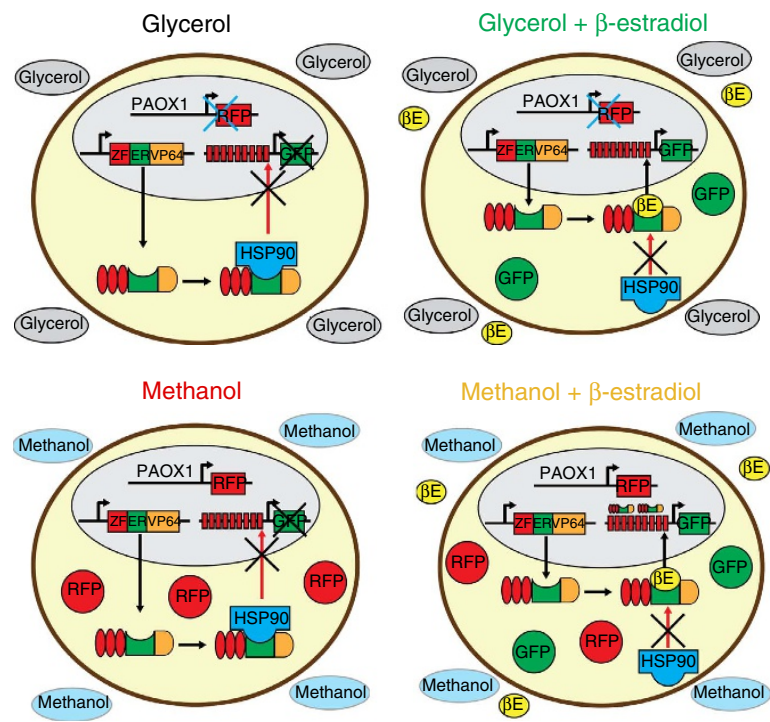

b
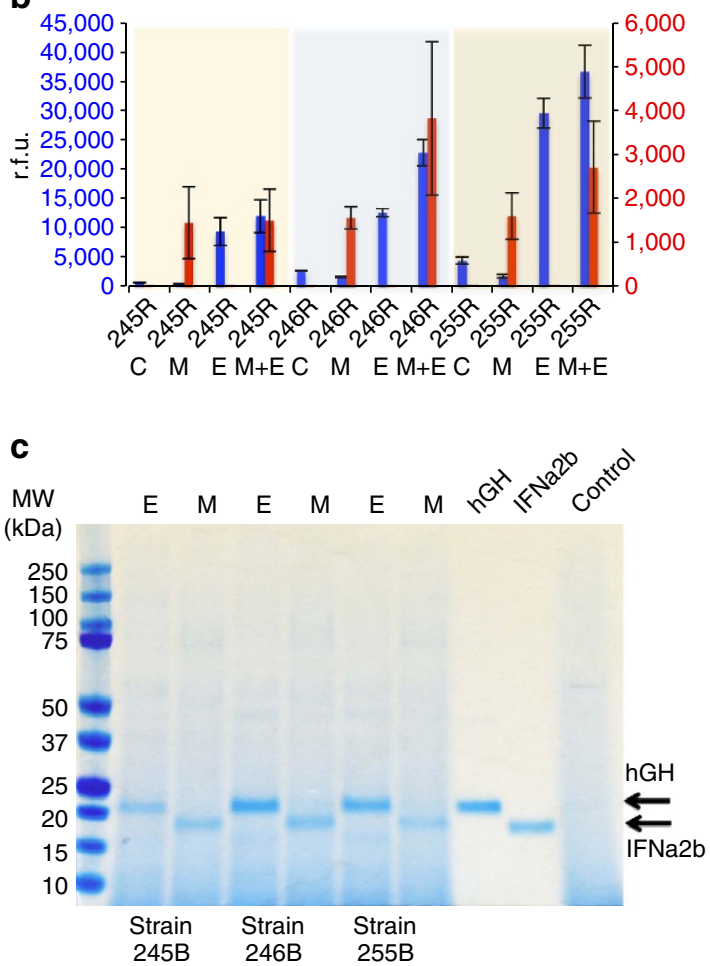

Figure 5 | Selectable biologics production from $\boldsymbol{P}$. pastoris strains with both an estradiol-inducible circuit and a methanol-inducible circuit. (a) Three strains containing circuit architectures with acceptable ON/OFF ratios were selected (245, 246 and 255) and added an RFP expression cassette controlled by the methanol-inducible AOX1 promoter (245R, 246R and 255R). (b) These strains expressed GFP when induced with $\beta$-estradiol in BMGY, expressed RFP when induced with BMMY and expressed both GFP and RFP when induced with $\beta$-estradiol in BMMY for 24 h. Error bars represent s.e.m. ( $n=3$ ). (c) Strains 245B, 246B and 255B were generated by replacing GFP and RFP in strains 245R, 246R and 255R with rHGH and IFN $\alpha 2 b$. Protein gel electrophoresis and Coomassie staining of supernatants from shake flasks demonstrated high-level expression of the desired biologic drug on stimulation for $24 \mathrm{~h}$ in BMGY for $\beta$-estradiol or BMMY for methanol.

important. For example, our current expression systems can achieve $\sim 110$-fold and $\sim 4$-fold ON:OFF ratios for IFN $\alpha 2 \mathrm{~b}$ and rHGH, respectively. However, concerns about background production of biologic A during induction of biologic $B$ and vice versa could make downstream purification processes and the regulatory approval process more challenging. Using recombinase-based switches to invert protein-expressing DNA cassettes $^{37,38}$ or additional repressors could reduce leakage beyond the transcriptional systems used here. For example, biologics-expressing cassettes could be surrounded by recombinase-recognition sites and initially encoded in inactive positions; expression of a recombinase could invert a targeted cassette and allow an upstream promoter to transcribe the correct messenger RNA. In addition, translational repressors or RNA interference could be used to further knock down undesirable expression levels in uninduced conditions. Combinatorial assembly of large numbers of genetic circuits followed by highthroughput screening for ones with enhanced ON:OFF ratios could further lower background expression. The integration of purification platforms with our biomanufacturing system could also help reduce background levels of biologics in uninduced states. To circumvent the limited number of inducible systems available for controlling biologics expression, future work could integrate more advanced gene circuits, such as multiplexers that enable a restricted set of $n$ inputs to control the expression of $2^{n}$ outputs. Additional inducible systems that leverage orthogonal chemical inputs or non-chemical inputs, such as light, could further help with the scalability of this system and, in the latter case, reduce logistics requirements.
Producing multi-component products such as vaccines could also circumvent the issue of undesirable background production, as multiple products could be expressed from a single strain. Furthermore, synthetic biology could help tailor a vaccine for specific populations, as different antigens are likely to be optimal in providing immunity depending on geographical location or timing. With artificial regulation over the expression of different antigens, we could control the ultimate formulation of multi-component vaccines on demand for optimal prophylaxis and mitigate concerns about background expression. These vaccines could be customized to specific outbreaks or local conditions to enhance their applicability. Currently, the production of such multi-component vaccines can require multiple manufacturing lines, each with its own FDA approval. A multiplexed expression platform for multi-component vaccines could conceivably reduce the regulatory burden for such products. Similarly, the controlled expression of multi-component biologic products could also benefit from this approach and help mitigate concerns over leaky background expression.

By combining genetic and hardware engineering, we have demonstrated a proof-of-concept, integrated and highly flexible platform for the programmable production of two biopharmaceuticals, IFN $\alpha 2 \mathrm{~b}$ and $\mathrm{rHGH}$. Future work includes scaling up the number of products that can be controllably manufactured, selecting specific drug candidates that are compatible with each other, enhancing production levels while reducing background expression levels and integrating this system with purification, polishing and analytics ${ }^{39}$. We envision that such technologies will enable the creation of small-scale, portable, fully integrated and 


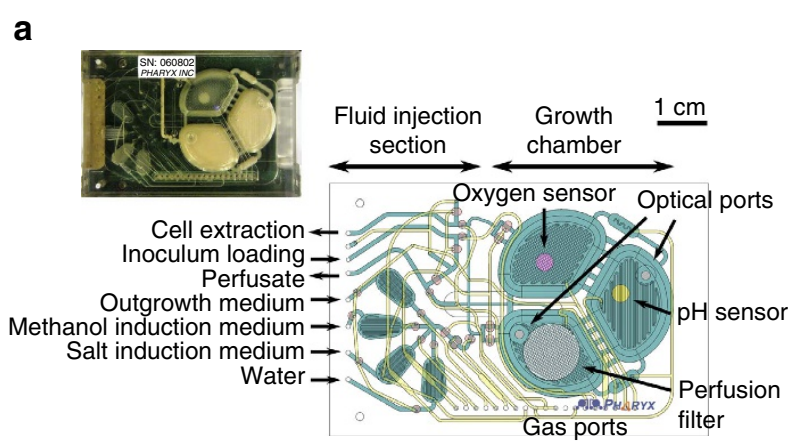

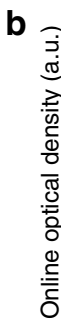

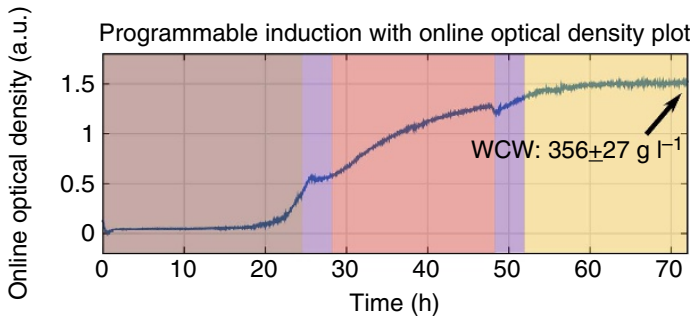

Outgrowth period

Medium switching

IFN $\alpha 2 b$ production rHGH production

C

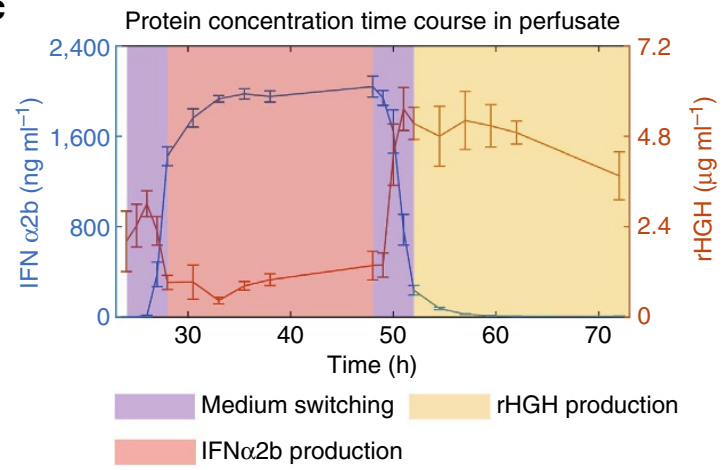

Figure 6 | Programmable protein production in a millilitre-scale table-top microbioreactor. (a) The principal component of the microbioreactor is a polycarbonate-PDMS membrane-polycarbonate sandwiched chip with active microfluidic circuits that are equipped for pneumatic routing of reagents, precise peristaltic injection, growth chamber mixing and fluid extraction. (b) Three-day continuous cultivation experiments for selectable production of two biologics were performed. The different operational phases are colour coded in the online OD plot for one experiment. The microbioreactor enabled high-density cell cultures up to a wet-cell weight (WCW) of $356 \pm 27 \mathrm{gl}^{-1}$. (c) Samples were collected the indicated time points and protein production was measured using ELISA, which demonstrated near single-dose drug production levels in fewer than $24 \mathrm{~h}$. Error bars represent s.e.m. $(n=4)$.

closed biomanufacturing systems that can advance the treatment of human diseases at the point-of-care.

\section{Methods}

Different media formulations namely BMGY, yeast extract peptone dextrose (YPD), BMMY, custom-made methanol medium and $\beta$-estradiol-containing salt medium were used in these experiments. BMGY medium contained $10 \mathrm{gl}^{-1}$ $(1 \%(\mathrm{w} / \mathrm{v}))$ yeast extract (VWR catalogue \#90004-092), $20 \mathrm{gl}^{-1}(2 \%(\mathrm{w} / \mathrm{v}))$ peptone (VWR catalogue \#90000-264), $100 \mathrm{mM}$ potassium phosphate monobasic (VWR catalog \#MK710012), $100 \mathrm{mM}$ potassium phosphate dibasic (VWR catalog \#97061$588), 4 \times 10^{-5} \%$ biotin (Life Technologies \#B1595), $13.4 \mathrm{gl}^{-1}$ (1.34\% (w/v)) Yeast Nitrogen Base (Sunrise Science catalogue \#1501-500) and 2\% glycerol (VWR catalogue \#AA36646-K7). YPD contained $1 \%$ yeast extract, $2 \%$ peptone and $2 \%$ dextrose (VWR catalogue \# BDH0230). BMMY contained $1 \%$ yeast extract, $2 \%$
Table 1 | Microbioreactor operation.

\begin{tabular}{llll} 
Time & Medium & Flow rate & Purpose \\
\hline $\mathrm{Oh}$ & $\mathrm{BMGY}$ & 0 & Outgrowth \\
14-24 $\mathrm{h}$ & BMGY & $0.5 \mathrm{ml} \mathrm{h}^{-1}$ & Outgrowth \\
$24-28 \mathrm{~h}$ & Methanol medium & $1.0 \mathrm{ml} \mathrm{h}^{-1}$ & Rapid medium switchover \\
$28-48 \mathrm{~h}$ & Methanol medium & $0.5 \mathrm{ml} \mathrm{h}^{-1}$ & IFN $\alpha 2 \mathrm{~b}$ production \\
$48-52 \mathrm{~h}$ & $\beta$-Estradiol medium & $1.0 \mathrm{ml} \mathrm{h}^{-1}$ & Rapid medium switchover \\
$52-72 \mathrm{~h}$ & $\beta$-Estradiol medium & $0.5 \mathrm{ml} \mathrm{h}^{-1}$ & rHGH production \\
\hline
\end{tabular}

BMGY, buffered glycerol-complex medium; IFN $\alpha 2 b$, interferon- $\alpha 2 b ; \mathrm{rHGH}$, recombinant human growth hormone.

Summary table including the microbioreactor operation timeline, inflow medium, flow rate and purpose for different operation phases in Fig. $6 \mathrm{~b}$.

peptone, $100 \mathrm{mM}$ potassium phosphate monobasic, $100 \mathrm{mM}$ potassium phosphate dibasic, $4 \times 10^{-5} \%$ biotin and $10 \mathrm{mll}^{-1}(1 \%(\mathrm{v} / \mathrm{v}))$ methanol (VWR catalogue \#VWRCBDH20864.4). The custom-made methanol medium contained $1.34 \%$ Yeast Nitrogen Base, $0.79 \mathrm{gl}^{-1}$ casaminoacids (Fisher \#BP1424-500), $2 \%$ methanol (VWR catalogue \#VWRCBDH20864.4) and 0.1\% antifoam 204 (Sigma-Aldrich catalogue \#A8311-50ML). The $\beta$-estradiol-containing salt medium contained $30 \mu \mathrm{M} \beta$-estradiol (Sigma-Aldrich catalogue \#E4389-100MG), $18.2 \mathrm{gl}^{-1} \mathrm{~K}_{2} \mathrm{SO}_{4}$ (VWR catalogue \#97062-578), $7.28 \mathrm{gl}^{-1} \mathrm{MgSO}_{4}$ (Sigma-Aldrich catalogue \#M7506-500G), $4.3 \mathrm{gl}^{-1} \mathrm{KOH}$ (Fisher \#P250-1), $0.08 \mathrm{gl}^{-1} \mathrm{CaSO}_{4} 2 \mathrm{H}_{2} \mathrm{O}$ (Sigma Aldrich catalogue \#C3771-500G), $13 \mathrm{mll}^{-1} 85 \%$ orthophosphoric acid (VWR catalogue \#E582-50ML), $1.47 \mathrm{gl}^{-1}$ sodium citrate (Fisher \#S279-500), $0.1 \%$ antifoam 204 and $\mathrm{pH}$ adjusted to 5.5 with ammonium hydroxide (Sigma-Aldrich catalogue \#318612-500ML). All individual reagents were prepared as stock solutions and mixed immediately before the experiments. BMGY, custom-made methanol medium and $\beta$-estradiol-containing salt medium were used in the experiment for initial outgrowth, IFN $\alpha 2 \mathrm{~b}$ production and $\mathrm{rHGH}$ production, respectively.

Plasmid construction. The multiple constructs used in these experiments were built using conventional restriction enzyme cloning and/or Gibson assembly using the vector pPICZ A (Invitrogen \#V190-20) as the backbone. All plasmids used in this manuscript are described in Supplementary Table 3 and have been deposited in the Addgene plasmid repository.

Strains. The wild-type Pichia pastoris strain NRRL Y- 11430, ATCC 76273 (reclassified as Komagataella phaffii) was used in this study.

Electroporation. Competent cells were prepared by first growing one single colony of $P$. pastoris in $5 \mathrm{ml} \mathrm{YPD}$ at $30^{\circ} \mathrm{C}$ overnight. Fifty microlitres of the resulting culture were inoculated in $100 \mathrm{ml}$ of YPD and grown at $30^{\circ} \mathrm{C}$ overnight again to an $\mathrm{OD}_{600} \sim 1.3-1.5$. The cells were then centrifuged at $1,500 \mathrm{~g}$ for $5 \mathrm{~min}$ at $4{ }^{\circ} \mathrm{C}$ and resuspended with $40 \mathrm{ml}$ of ice-cold sterile water, centrifuged at $1,500 \mathrm{~g}$ for $5 \mathrm{~min}$ at $4^{\circ} \mathrm{C}$ and resuspended with $20 \mathrm{ml}$ of ice-cold sterile water, centrifuged at $1,500 \mathrm{~g}$ for $5 \mathrm{~min}$ at $4{ }^{\circ} \mathrm{C}$ and resuspended in $20 \mathrm{ml}$ of ice-cold $1 \mathrm{M}$ sorbitol, and centrifuged at $1,500 \mathrm{~g}$ for $5 \mathrm{~min}$ at $4{ }^{\circ} \mathrm{C}$ and resuspended in $0.5 \mathrm{ml}$ of ice-cold $1 \mathrm{M}$ sorbitol. For transformation of the landing pad plasmids, $80 \mu \mathrm{l}$ of competent cells were mixed with 5-20 $\mu \mathrm{g}$ of linearized DNA and transferred to an ice-cold $0.2 \mathrm{~cm}$ electroporation cuvette for $5 \mathrm{~min}$. For recombinase-based transformations, $10 \mu \mathrm{g}$ of circular transfer vector and $10 \mu \mathrm{g}$ of circular recombinase expression vector were combined, added to $80 \mu \mathrm{l}$ of competent cells and incubated for $5 \mathrm{~min}$ in an ice-cold $0.2 \mathrm{~cm}$ electroporation cuvette. Pulse parameters were $1,500 \mathrm{~V}, 200 \Omega$ and $25 \mu \mathrm{F}$. Immediately after pulsing, $1 \mathrm{ml}$ of ice-cold $1 \mathrm{M}$ sorbitol was added to the cuvette and the cuvette content was transferred to a sterile culture tube containing $2 \times$ YPD. The culture tubes were incubated for $2 \mathrm{~h}$ at $30^{\circ} \mathrm{C}$ with shaking and $50-100 \mu \mathrm{l}$ of the culture was spread on Yeast Extract Peptone Dextrose Sorbitol (YPDS) plates ( $1 \%$ yeast extract, $2 \%$ peptone, $1 \mathrm{M}$ sorbitol, $1 \%$ dextrose and $2 \%$ agar) with the appropriate selection antibiotic (zeocin $100 \mu \mathrm{g} \mathrm{ml}^{-1}$; G418 $100 \mu \mathrm{g} \mathrm{ml}^{-1}$ ).

Cell induction and flow cytometry. One single colony was grown in $1 \mathrm{ml}$ of BMGY in a $12-\mathrm{ml}$ culture tube at $30^{\circ} \mathrm{C}$ in a shaking incubator (250-300 r.p.m.) overnight. The cells were centrifuged at $500 \mathrm{~g}$ for $5 \mathrm{~min}$ at room temperature and washed twice with PBS. After the second wash, the cells were resuspended in induction medium consisting of BMMY or BMGY with $\beta$-estradiol. After $24 \mathrm{~h}$, the cultures were centrifuged at $500 \mathrm{~g}$ for $5 \mathrm{~min}$ at room temperature, washed with $1 \mathrm{ml}$ of PBS twice, resuspended in $1 \mathrm{ml}$ of PBS and used for flow cytometry with a LSR Fortessa cell analyser.

ELISA assay. The concentration of hGH and IFN- $\alpha 2 b$ in each of the samples was determined by ELISA assay. Solid-phase 96-well ELISA plates were used, 
Table 2 | Microbioreactor production.

\begin{tabular}{|c|c|c|c|c|}
\hline IFN $\alpha 2 b$ & rHGH & IFN $\alpha \mathbf{2 b}$ & rHGH & Wet cell weight \\
\hline Production (20 h) & Production $(20 \mathrm{~h})$ & Leakage $(20 \mathrm{~h})$ & Leakage $(20 \mathrm{~h})$ & \\
\hline $19.73 \pm 0.72 \mu \mathrm{g}$ & $43.7 \pm 6.3 \mu \mathrm{g}$ & $0.177 \pm 0.032 \mu \mathrm{g}$ & $10.8 \pm 2.9 \mu \mathrm{g}$ & $356 \pm 27 \mathrm{gl}^{-1}$ \\
\hline
\end{tabular}

specifically designed for the quantification of hGH (Quantikine ELISA, R\&D Systems) and IFN- $\alpha$ (Verikine Human IFN-alpha ELISA Kit, PBL Assay Science). The products were provided with the proper standard stock solutions or powder for each of the assays. For hGH, the standard curve was calculated using concentrations ranging between 3,200 and $25 \mathrm{gg} \mathrm{ml}^{-1}$, in twofold serial dilutions. For IFN $\alpha 2 b$, the extended range standard curve was used, with an additional large concentration point: the standard concentration varied between 156 and $10,000 \mathrm{\rho} \mathrm{ml}^{-1}$, in twofold serial dilutions. For both assays, negative controls were also included. Before the protein quantification, the samples were diluted in the appropriate buffer so that the concentrations determined would fall within the assay and equipment limits.

The optical density values obtained for each of the assays were plotted using a four-parameter fit for the standard curve. Each sample was measured twice and the results represent the average and s.d. of three biological replicates.

Quantitative PCR. Genomic DNA from different strains was isolated using the YeaStar Genomic DNA kit (Zymo) and the resulting preps were diluted down to a concentration of $5 \mathrm{ngll}^{-1}$. qPCR mixtures were prepared using the LightCycler 480 SYBR Green Master mix (Roche) with $10 \mathrm{ng}$ genomic DNA from each strain was used with $400 \mathrm{nM}$ of each primer per assay in a total reaction volume of $20 \mu \mathrm{l}$. Reactions were performed in LightCycler 480 96-well reaction plates in triplicate with a standard curve for each gene generated from serial ten-fold dilutions of $2 \mathrm{ng}$ of plasmid DNA containing the gene-of-interest. The amplification conditions were as follows: $95^{\circ} \mathrm{C}$ for $10 \mathrm{~min}$ followed by 45 cycles of $95^{\circ} \mathrm{C}$ for $10 \mathrm{~s}, 56^{\circ} \mathrm{C}$ for $15 \mathrm{~s}$ and $72{ }^{\circ} \mathrm{C}$ for $20 \mathrm{~s}$. The amplification period was followed by a melting curve analysis with a temperature gradient of $0.1^{\circ} \mathrm{C} \mathrm{s}^{-1}$ from $65^{\circ} \mathrm{C}$ to $95^{\circ} \mathrm{C}$. An amplicon from the single-copy GAPDH or ACT1 genes was used for normalization. Using the published genome size of $9.4 \mathrm{Mbp}$, we expected 98,000 copies of the genome to be present in $1 \mathrm{ng}$ haploid P. pastoris genomic DNA. Absolute copy number for the gene-of-interest in each strain was calculated using the mean $\mathrm{Ct}$ value and the corresponding standard curve.

The sequence of the primers used were as follows: hGH ( $5^{\prime}$-GGGCAGATCTT CAAGCAGAC- $3^{\prime}, 5^{\prime}$-CTCGACCTTGTCCATGTCCT- $\left.3^{\prime}\right)$; IFN (5' -TTCCCACA AGAGGAATTTGG-3', $5^{\prime}$-AGGCTGCTGAGGAATCTTTG-3'); GAPDH (5'-TGG GTTACACTGAAGATGCC- $3^{\prime}, 5^{\prime}$-CGTTGTCGTACCAAGAGATCAG- $3^{\prime}$ ); ACT1 (5'-TGGTATCGTTTTGGACTCTGG- $3^{\prime}, 5^{\prime}$-AGCGTGTGGTAAGGAGAAAC- $\left.3^{\prime}\right)$; and landing pad (5'-TGTCTTCGTGGTTTGTCTGG-3', 5'-TCTTGTAGTTGCC GTGTCG-3').

Microbioreactor. Parts for the microbioreactor experiment were purchased from Pharyx Inc. A single bioreactor control hub (Pharyx, \#MBS-004) with an overall footprint of $31 \mathrm{~cm} \mathrm{(w)} \times 34 \mathrm{~cm} \mathrm{(d)} \times 36 \mathrm{~cm}$ (h) was used to control four independent microbioreactor units (Pharyx, \#MCM-001) for the fermentation experiment. Each microbioreactor unit interfaces with a single-use disposable microfluidic chip made of a sandwiched polycarbonate-PDMS membranepolycarbonate structure (Pharyx, \#CCPST-1) to carry out peristaltic control and online sensing. The design, fabrication and system configuration for the microbioreactor have been described previously 33,40 . To provide the rapid medium changeover required for this study, a perfusion filter (Pall Corp., Supor 800, \#60109) was incorporated into the cultivation chamber ${ }^{41}$ to allow medium flow-through, while maintaining cells inside the growth chamber.

Microbioreactor flow modelling. To account for the microbioreactor media concentration dynamics during the changeover period, a simple model based on fluid concentration rate equation under equal inflow and outflow rate was constructed. For simplicity, cellular consumption on media material was not taken into account in this model. Assuming that $S(t)$ represents a particular medium concentration in the growth chamber, $S_{\text {in }}(t)$ represents the concentration of this medium in the input flow, $F$ represents the input flow rate and $V$ represents the growth chamber volume, then the rate equation for the medium concentration in the growth chamber can be modelled as

$$
\frac{\partial S(t)}{\partial t}=\frac{F}{V}\left[S_{\text {in }}(t)-S(t)\right] .
$$

For the situation of introducing a completely new medium into the growth chamber to replace an old medium similar to our programmable biologics production experiment, assuming $S_{\text {in }}(t)$ is constant during the time of changeover and $t=0$ being the start of changeover event, the new medium concentration can be simply solved analytically as

$$
S_{\text {new }}(t)=S_{\text {in }}\left(1-e^{-\frac{F}{V} t}\right) .
$$

Meanwhile, the concentration of the old medium due to flush out follows as

$$
S_{\text {old }}(t)=S_{\text {old }} e^{-\frac{F}{V} t},
$$

assuming $S_{\text {old }}$ is the concentration of the old medium before the changeover event. Therefore, a flow rate of $1 \mathrm{ml} \mathrm{h}^{-1}$ for the changeover period of $4 \mathrm{~h}$ would result in a medium replacement percentage of $\sim 1-e^{-4} \approx 98.2 \%$. In contrast, if the changeover rate stays as $0.5 \mathrm{ml} \mathrm{h}^{-1}$ similar to the case for the production period, the medium replacement percentage would be $\sim 1-e^{-2} \approx 86.5 \%$, where there would still be a substantial amount of the previous medium leftover after the changeover event. Such physical modelling of the microbioreactor operation provides a simple yet effective design guideline to complement the operational flexibility of our manufacturing platform.

Microbioreactor experiment. The microbioreactor chips were $\gamma$-irradiated and sealed as part of the standard pre-inoculation sterile protocol. The medium bottles and feed lines were autoclaved separately. The initial inoculum was loaded from a single colony from a YPD plate stored at $4{ }^{\circ} \mathrm{C}$ at $0 \mathrm{~h}$. The fermentation parameter plot for the online $\mathrm{OD}$, dissolved oxygen, $\mathrm{pH}$ and temperature for one experiment is shown in Supplementary Fig. 9. As described in the manuscript, the fermentation temperature was controlled at $30 \pm 0.1{ }^{\circ} \mathrm{C}$ throughout the entire experiment. The dissolved oxygen level of the microbioreactor was controlled by dynamically changing the gas feed line between air and oxygen to match the dissolved oxygen set point, which was set to $100 \%$ air saturation in the experiment. As the cells grow and the overall oxygen consumption rate increases, the gas controller gradually increases the oxygen content in the gas feed line to maintain the dissolved oxygen set point. Once the cell oxygen consumption rate overpasses the oxygen transfer rate by pure oxygen supply, the dissolved oxygen drops below the set point and the supply gas remains at $100 \%$ oxygen. The online OD is monitored through light scattering across an optical path length of $\sim 250 \mu \mathrm{m}$ inside the growth chamber with a 630-nm light-emitting diode. The linear response range for this $\mathrm{OD}$ sensor is around $0 \sim 0.7$ online $\mathrm{OD}$ unit. Above $\sim 0.7$ online $\mathrm{OD}$ unit, the sensor reading no longer increases linearly with the cell density. The online $\mathrm{pH}$ data are also recorded during the fermentation process. The $\mathrm{pH}$ sensor is rated for $\mathrm{pH}$ values of $5.5 \sim 8.5$. During the third day, the $\mathrm{pH}$ reading falls below 5.5 and therefore may not accurately represent the actual $\mathrm{pH}$ of the culture environment. Over the course of the study, perfusate samples were collected downstream of the perfusion filter and were stored at $4{ }^{\circ} \mathrm{C}$ before processing with ELISA assay.

Additional microbioreactor runs. In addition to the microbioreactor run presented in the body manuscript, three additional runs of the microbioreactor experiments were performed over the course of 5 months. Each run is marked by the time of the experiment and consists of two independent microbioreactors operating the same protocol in parallel. The protein concentration time course plot is shown in Supplementary Fig. 10 and the cumulative protein production quantity is summarized in Supplementary Table 4. Overall, the same switching behaviour was observed across all runs with some run-to-run protein production variations observed, especially for the December 2014 Run, where rHGH production was much higher than the rest. These run-to-run variations are probably caused by our experimentation with the inner surface coating and $\gamma$-irradiation protocol of the microbioreactor chip. After the microbioreactor parts were fabricated and bonded, an inner surface coating protocol with polyethylene glycol (PEG)-silane treatment was carried out, followed by a chip nitrogen purge and bagging procedure, and $\gamma$-irradiation sterilization. Some experimentation on this protocol in terms of PEG-silane treatment time and chip nitrogen purge procedure in search for the optimal coating condition was carried across the early microbioreactor runs. For all runs, the chips used in the same run were fabricated with exactly the same protocol and therefore do not cause much variation within each microbioreactor run. As we standardized our chip fabrication protocol during this 5-month time period, our run-to-run variations reduced substantially (the March 2015 Run and the main body run that happened in June 2015 had much less production variations compared with the rest). Nonetheless, the result from the March 2015 Run suggests the potential of high production yield comparable to industrial level for our 
manufacturing platform with suitable manufacturing standardization and bioprocess optimization.

Data availability. The data that support the findings of this study are available from the corresponding author upon request. The plasmids used in this manuscript have been deposited in Addgene. Addgene accession numbers can be found in Supplementary Table 3.

\section{References}

1. Thiel, K. A. Biomanufacturing, from bust to boom...to bubble? Nat. Biotechnol. 22, 1365-1372 (2004)

2. Dove, A. Uncorking the biomanufacturing bottleneck. Nat. Biotechnol. 20, 777-779 (2002).

3. Gottschalk, U., Brorson, K. \& Shukla, A. A. The need for innovation in biomanufacturing. Nat. Biotechnol. 30, 489-492 (2012).

4. Farid, S. S., Thompson, B. \& Davidson, A. in 10th Annual bioProcess UK Conference, MAbs 6, 1357-1361 (3-4, December 2013, London, UK, 2014).

5. Hwang, T. J. Stock market returns and clinical trial results of investigational compounds: an event study analysis of large biopharmaceutical companies. PLoS ONE 8, e71966 (2013).

6. Ledford, H. First biosimilar drug set to enter US market. Nature 517, 253-254 (2015).

7. Siganporia, C. C., Ghosh, S., Daszkowski, T., Papageorgiou, L. G. \& Farid, S. S. Capacity planning for batch and perfusion bioprocesses across multiple biopharmaceutical facilities. Biotechnol. Prog. 30, 594-606 (2014).

8. Willke, R. J. et al. Melding regulatory, pharmaceutical industry, and U.S. payer perspectives on improving approaches to heterogeneity of treatment effect in research and practice. Value Health 16, S10-S15 (2013).

9. Zhu, J. Mammalian cell protein expression for biopharmaceutical production. Biotechnol. Adv. 30, 1158-1170 (2012).

10. Goldrick, S., Stefan, A., Lovett, D., Montague, G. \& Lennox, B. The development of an industrial-scale fed-batch fermentation simulation. J. Biotechnol. 193, 70-82 (2015).

11. Diel, B., Manzke, C. \& Peuker, T. Flexible biomanufacturing processes that address the needs of the future. Adv. Biochem. Eng. Biotechnol. 138, 207-237 (2014).

12. Berlec, A. \& Strukelj, B. Current state and recent advances in biopharmaceutical production in Escherichia coli, yeasts and mammalian cells. J. Ind. Microbiol. Biotechnol. 40, 257-274 (2013).

13. Mattanovich, D. et al. Recombinant protein production in yeasts. Methods Mol. Biol. 824, 329-358 (2012).

14. Vervecken, W. et al. In vivo synthesis of mammalian-like, hybrid-type N-glycans in Pichia pastoris. Appl. Environ. Microbiol. 70, 2639-2646 (2004).

15. Vervecken, W., Callewaert, N., Kaigorodov, V., Geysens, S. \& Contreras, R. Modification of the N-glycosylation pathway to produce homogeneous, human-like glycans using GlycoSwitch plasmids. Methods Mol. Biol. 389, 119-138 (2007).

16. Zhang, N. et al. Glycoengineered Pichia produced anti-HER2 is comparable to trastuzumab in preclinical study. MAbs 3, 289-298 (2011).

17. Macauley-Patrick, S., Fazenda, M. L., McNeil, B. \& Harvey, L. M. Heterologous protein production using the Pichia pastoris expression system. Yeast 22, 249-270 (2005).

18. Vogl, T., Hartner, F. S. \& Glieder, A. New opportunities by synthetic biology for biopharmaceutical production in Pichia pastoris. Curr. Opin. Biotechnol. 24, 1094-1101 (2013).

19. Prielhofer, R. et al. Induction without methanol: novel regulated promoters enable high-level expression in Pichia pastoris. Microb. Cell Fact. 12, 5 (2013).

20. Koller, A., Valesco, J. \& Subramani, S. The CUP1 promoter of Saccharomyces cerevisiae is inducible by copper in Pichia pastoris. Yeast 16, 651-656 (2000).

21. Resina, D., Cos, O., Ferrer, P. \& Valero, F. Developing high cell density fed-batch cultivation strategies for heterologous protein production in Pichia pastoris using the nitrogen source-regulated FLD1 promoter. Biotechnol. Bioeng. 91, 760-767 (2005).

22. Cereghino, J. L. \& Cregg, J. M. Heterologous protein expression in the methylotrophic yeast Pichia pastoris. FEMS Microbiol. Rev. 24, 45-66 (2000).

23. Yamaguchi, S. et al. A method for producing transgenic cells using a multi-integrase system on a human artificial chromosome vector. PLoS ONE 6, e17267 (2011).

24. Khalil, A. S. et al. A synthetic biology framework for programming eukaryotic transcription functions. Cell 150, 647-658 (2012).

25. McIsaac, R. S. et al. Synthetic gene expression perturbation systems with rapid, tunable, single-gene specificity in yeast. Nucleic Acids Res. 41, e57 (2013).

26. Perez-Pinera, P. et al. Synergistic and tunable human gene activation by combinations of synthetic transcription factors. Nat. Methods 10, 239-242 (2013).
27. Polstein, L. R. \& Gersbach, C. A. Light-inducible spatiotemporal control of gene activation by customizable zinc finger transcription factors. J. Am. Chem. Soc. 134, 16480-16483 (2012).

28. Day, C. D. et al. Transgene integration into the same chromosome location can produce alleles that express at a predictable level, or alleles that are differentially silenced. Genes Dev. 14, 2869-2880 (2000).

29. Ramirez, A. et al. Sequence and chromosomal context effects on variegated expression of keratin $5 /$ lac $Z$ constructs in stratified epithelia of transgenic mice. Genetics 158, 341-350 (2001).

30. Chen, M. T. et al. Generation of diploid Pichia pastoris strains by mating and their application for recombinant protein production. Microb. Cell Fact. 11, 91 (2012).

31. Degelmann, A. et al. Strain and process development for the production of human cytokines in Hansenula polymorpha. FEMS Yeast Res. 2, 349-361 (2002).

32. Law, V. et al. DrugBank 4.0: shedding new light on drug metabolism. Nucleic Acids Res. 42, D1091-D1097 (2014).

33. Lee, K. S., Boccazzi, P., Sinskey, A. J. \& Ram, R. J. Microfluidic chemostat and turbidostat with flow rate, oxygen, and temperature control for dynamic continuous culture. Lab Chip 11, 1730-1739 (2011).

34. Clincke, M. F. et al. Very high density of CHO cells in perfusion by ATF or TFF in WAVE bioreactor. Part I. Effect of the cell density on the process. Biotechnol. Prog. 29, 754-767 (2013).

35. Drugs.com. http://www.drugs.com/dosage/nutropin-aq.html (2015).

36. Bareither, R. \& Pollard, D. A review of advanced small-scale parallel bioreactor technology for accelerated process development: current state and future need. Biotechnol. Prog. 27, 2-14 (2011).

37. Siuti, P., Yazbek, J. \& Lu, T. K. Synthetic circuits integrating logic and memory in living cells. Nat. Biotechnol. 31, 448-452 (2013).

38. Yang, L. et al. Permanent genetic memory with $>1$-byte capacity. Nat. Methods 11, 1261-1266 (2014).

39. Warikoo, V. et al. Integrated continuous production of recombinant therapeutic proteins. Biotechnol. Bioeng. 109, 3018-3029 (2012).

40. Lee, K. S. \& Ram, R. J. Plastic-PDMS bonding for high pressure hydrolytically stable active microfluidics. Lab Chip 9, 1618-1624 (2009).

41. Mozdzierz, N. J. et al. A perfusion-capable microfluidic bioreactor for assessing microbial heterologous protein production. Lab Chip 15, 2918-2922 (2015).

\section{Acknowledgements}

We thank Dr Christopher Love for feedback on the paper. The project or effort depicted was or is also sponsored by the Defense Advanced Research Projects Agency. The content of the information does not necessarily reflect the position or the policy of the Government.

\section{Author contributions}

P.P.P., N.H., S.C., J.C., O.P, K.S., K.L., R.R. and T.K.L. designed experiments. P.P.P., N.H., S.C., J.C., O.P., K.L. and K.S. performed experiments. P.P.P., N.H., S.C., J.C., O.P., K.S., K.L., R.R. and T.K.L. analysed the data. P.P.P., N.H., S.C., J.C., O.P., K.S., R.R. and T.K.L. wrote the manuscript.

\section{Additional information}

Supplementary Information accompanies this paper at http://www.nature.com/ naturecommunications

Competing financial interests: The authors declare no competing financial interest.

Reprints and permission information is available online at http://npg.nature.com/ reprintsandpermissions/

How to cite this article: Perez-Pinera, P. et al. Synthetic biology and microbioreactor platforms for programmable production of biologics at the point-of-care. Nat. Commun. 7:12211 doi: 10.1038/ncomms12211 (2016).

This work is licensed under a Creative Commons Attribution 4.0 International License. The images or other third party material in this article are included in the article's Creative Commons license, unless indicated otherwise in the credit line; if the material is not included under the Creative Commons license, users will need to obtain permission from the license holder to reproduce the material. To view a copy of this license, visit http://creativecommons.org/licenses/by/4.0/ 